\title{
Acceleration time in the aorta and pulmonary artery measured by Doppler echocardiography in the midtrimester normal human fetus
}

Sir,

I wish to take issue with Machado et al (1987;58: 15-8) who measured aortic and pulmonary artery acceleration times in a series of human fetuses and inferred that pulmonary vascular resistance was increased. They based this conclusion on studies in adults that showed that acceleration time is shortened in the pulmonary trunk when pulmonary mean pressure is increased. ${ }^{1}$ Machado et al inferred that the fetal pulmonary vascular resistance is higher because acceleration times were slightly shorter in the fetal pulmonary trunk than the aorta.

The observation that flow profiles in the aortic and pulmonary circuits are different is not new; it was reported in the fetuses of sheep by Rudolph. ${ }^{2}$ It is well known that aortic and pulmonary pressures are equal in the fetus and that fetal pulmonary vascular resistance is high. The precise relation of the vascular resistances of the aortic and pulmonary circuits has, to my knowledge, not been investigated in any animal model. Because the ductus is widely patent in utero the vascular resistance reflected by events in the pulmonary trunk, where Machado et al made their measurement, must have reflected the vascular resistance of the placenta (which is low) and that of the lower body of the fetus as well.

The original observation by Kitabatake et al assumes that acceleration time relates to mean pulmonary pressure and consequently vascular resistance. ${ }^{1}$ There are other more complex factors which alter this measurement, including the vascular impedance, capacitance of the vascular bed in the distal circuit, the intrinsic contractile properties of the left and right ventricles ${ }^{3}$ and the preload conditions of the two ventricles in the fetus. These factors may be quite different in the fetus and adult, and Machado et al's inference that the acceleration time reflects higher pulmonary vascular resistance seems to be too simple.

It is well known that pulmonary vascular resistance in the fetus is high although pulmonary and aortic pressures are similar. Machado et al provide evidence only that there is a slight difference in acceleration times, with that of the pulmonary trunk measurement being shorter, without examining the factors affecting acceleration time which appear to be complex and affected by many physiological variables.

Norman H Silverman,

Pediatric Echocardiography Laboratory,

University of California,

San Francisco, California 94143, USA.

\section{References}

1 Kitabatake A, Inoue $M$, Asao $M$, et al. Non-invasive evaluation of pulmonary hypertension by pulsed Doppler technique. Circulation 1983;68:302-9.

2 Rudolph AM. Organization and control of the fetal circulation. In: Jones CT, Nathanielsz PW, eds. The physiological development of the fetus and newborn. London: Academic Press, 1985:343-53.

3 Reller MD, Morton MJ, Reid DL, Thornburg KL. Fetal lamb ventricles respond differently to filling and arterial pressures and to in utero ventilation. Pediatr Res 1987;22:621-6.

This letter was shown to the authors, who reply as follows:

Sir,

Dr Silverman seems to have misunderstood the intention of our paper. Apparently he believes that we are saying something about the lung fields. But in the fetus the pressure in the main pulmonary artery reflects the cumulative resistance of the lung fields, the lower body of the fetus, and the placenta.

We found that the acceleration time in the pulmonary artery in the early human fetus was different from that in the aorta. Based on evidence from adults and children we felt this indicated a difference between the pressures in the two great arteries. We agree with Dr Silverman that alternative indices of cardiovascular function may account for our findings. Those he quotes are not measurable in the intact human fetus. Our interpretation is supported by further work by us that shows that later in pregnancy the acceleration times in both great arteries become equal; this is consistent with the fall in placental resistance as gestation advances. Conversely, when placental resistance is abnormally high the acceleration time in the pulmonary artery is abnormally short in late pregnancy. 